\title{
Análise da distribuição da cobertura vegetal de Áreas Verdes Públicas em Governador Valadares, Minas Gerais, Brasil
}

\author{
Analysis of vegetation cover distribution in Public Green Areas in Governador \\ Valadares, Minas Gerais State, Brazil
}

\author{
Antônio Carlos de Oliveira Martins Júnior ${ }^{1}$ \\ Sarah Louback Reis da Cunha ${ }^{2}$ \\ Rebeca Soares Quintão Borges ${ }^{4}$ \\ Renata Bernardes Faria Campos ${ }^{4}$ \\ Estêvão Preisigke Seidler Gaede \\ Matheus Louback Reis da Cunha ${ }^{6}$ \\ Pollyana Candido da Silva Martins"
}

\begin{abstract}
Palavras-chave:
Ecologia urbana

Indicadores ambientais urbanos

Planejamento urbano.

Resumo

O crescimento da população urbana no Brasil deve continuar até 2050, afetando a relação entre os habitantes e as áreas verdes urbanas, cuja vegetação oferece benefícios ao ambiente e à saúde humana. Essas áreas devem ser conservadas e podem ser monitoradas utilizando técnicas e indicadores de medição e distribuição, relacionando sua área e cobertura vegetal (CV) com a população urbana. O estudo mediu e avaliou a distribuição da CV de Áreas Verdes Públicas (AVPs) da cidade de Governador Valadares, Minas Gerais, Brasil. Foram utilizadas uma ortofotografia e o software AutoCad ${ }^{\mathrm{R})}$ para a medição da CV e foram calculados e classificados os índices de AVP e CV-AVP para cidade e bairros. Analisou-se a relação de CV com a área da AVP e o zoneamento urbano e dos índices com a idade dos bairros. A proporção de CV é inconstante para a área de AVP e zonas urbanas mais populosas apresentaram menor CV. Nos bairros as AVPs que possuem tamanho recomendado não apresentaram CV satisfatória e a maioria dos índices foram nulos. As AVPs não têm sido mantidas com predomínio de CV, indicando limitação dos serviços ecológicos da vegetação para a população urbana. Portanto, é preciso alternativas de espaços verdes em regiões antigas e sem AVPs, conservar AVPs com CV satisfatória e reflorestar as que possuem CV insatisfatória. A CV urbana foi medida em uma ortofotografia de 0,1 pixell cm, indicando índices de AVP e CV-AVP e o mapa de distribuição da CV de AVPs como ferramentas adequadas e de baixo custo para o planejamento urbano, visando os serviços ambientais que a vegetação oferece à população.
\end{abstract}

\footnotetext{
${ }^{1}$ Universidade Federal do Rio Grande do Sul, PPG em Recursos Hídricos e Saneamento Ambiental, Porto Alegre, RS, Brasil. antoniocomjr@gmail.com

${ }^{2}$ Prefeitura Municipal de Governador Valadares, SMOSU, Governador Valadares, MG, Brasil. louback.rc@ gmail.com

${ }^{3}$ Universidade Vale do Rio Doce, PPG em Eficiência e Desempenho de Edificações Habitacionais, Governador Valadares, MG,

Brasil. rebecaborges.eng@gmail.com

${ }^{4}$ Universidade Vale do Rio Doce, PPG em Gestão Integrada do Território, Governador Valadares, MG, Brasil.

rbfcampos@gmail.com

${ }^{5}$ Instituto Federal de Minas Gerais, PPG em Engenharia de Segurança do Trabalho, Governador Valadares, MG, Brasil.

estevaogaede@hotmail.com

${ }^{6}$ Universidade Vale do Rio Doce, Núcleo de Ciências e Tecnologia, Governador Valadares, MG, Brasil.

louback12@hotmail.com

${ }^{7}$ Universidade Vale do Rio Doce, Núcleo de Ciências e Tecnologia, Governador Valadares, MG, Brasil.

pollyana.cs@hotmail.com
} 


$\begin{array}{ll}\text { Keywords: } & \text { Abstract } \\ \text { Urban ecology } & \text { Urban population growth in Brazil is expected to remain on rising until } \\ \text { Urban environmental indicators } & \text { 2050, and it will affect the relationship between city residents and urban } \\ \text { Urban planning } & \text { green areas, whose vegetation favor the environment and human health. } \\ \text { These areas must be conserved and they can be monitored through the } & \text { application of techniques and through measurement and distribution } \\ & \text { indicators by relating their area and vegetation cover (VC) to the urban } \\ \text { population. VC distribution in Public Green Areas (PGA) in Governador } & \text { Valadares City, Minas Gerais State, Brazil, was measured and assessed. } \\ \text { An orthophoto and the AutoCad }{ }^{(\mathrm{R})} \text { software were used to measure VC and } \\ \text { to calculate and classify PGA and PGAVC indices for the city and its } \\ \text { neighborhoods. Also, the association between VC and PGA area, and } \\ \text { urban zoning, as well as between indices and neighborhoods' age, were } \\ \text { analyzed. VC ratio in the PGA area is inconstant; moreover, more } \\ \text { populated zones presented less VC. PGAs presenting the recommended } \\ \text { size did not present satisfactory VC in the neighborhoods and most } \\ \text { indices recorded value equal to zero. PGAs did not remain the same due } \\ \text { to VC prevalence, and it may limit vegetation's ecological services to the } \\ \text { urban population. Therefore, alternative green spaces are needed in old } \\ \text { regions without PGAs, PGA conservation in regions with satisfactory VC, } \\ \text { as well as it is essential reforesting areas recording unsatisfactory VC. } \\ \text { Urban VC was measured in an orthophoto (0.1 pixel/cm) and it indicated } \\ \text { that PGA and PGAVC indices and PGAs VC distribution map are } \\ \text { suitable and low־cost urban planning tools aimed at environmental } \\ \text { services offered by vegetation to the population. }\end{array}$

\section{INTRODUÇÃO}

A população urbana no Brasil continuará crescendo até 2050 (UN, 2018) e a direção e força desse processo de expansão podem afetar a relação entre a população e as áreas verdes urbanas e, consequentemente, os serviços ecológicos oferecidos por estas às pessoas (FEIBER, 2004; TZOULAS et al., 2007).

Por isso, essas áreas não têm sido apenas um atributo ornamental, mas também uma exigência legal para a conservação do ambiente urbano. A Lei 12.651/2012 define área verde como espaços onde exista predominância de vegetação nativa, natural ou recuperada e seja livre de construções de moradias (BRASIL, 2012). Estudos consideraram praças públicas, áreas com cobertura vegetal situadas no meio urbano, entre outras definições (CAVALHEIRO; NUCCI, 1998; BOLDRIN et al., 2016). Apesar da diversidade conceitual, a cobertura vegetal é inerente às áreas verdes urbanas, sendo aquela a responsável pelos serviços ecológicos que chegam à população.

Nucci e Cavalheiro (1999) citam funções da cobertura vegetal: estabilização de superfícies, obstáculo contra o vento, proteção da qualidade da água, filtração do ar e equilíbrio do índice de umidade, fornecimento de alimentos, contribuição na preservação das nascentes e mananciais, valorização visual e ornamental e redução de ruídos e partículas de poeiras em suspensão.

Elementos naturais, incluindo a vegetação, melhoram a vida nas cidades e constituem relações diretas com a saúde humana (BONZI, 2017; SOLTANIFARD; JAFARI, 2019). "A falta de conexão com a natureza acarreta inúmeros problemas psicológicos, como aumento do estresse e o transtorno de déficit de atenção e hiperatividade" (FARR, 2013, p.36). Especificamente, o enfrentamento da pandemia do coronavírus, responsável pela COVID-19, destaca a importância do verde e de seus benefícios físicos e mentais (SANTOS, 2020). O convívio social em áreas externas, principalmente nesses espaços, é benéfico e pode ser seguro se mantidas as regras de distanciamento e proteção individual, além do ar livre dispersar a carga viral e expor as partículas a diversos fatores climáticos (GUADAGNIN, 2020).

Visando todas essas atribuições, é necessário que as áreas verdes urbanas sejam vegetadas (GUZZO; CARNEIRO; OLIVEIRA JÚNIOR, 2006) e tenham pelo menos $70 \%$ de sua superfície em solo permeável e livre de edificações (BOLDRIN et al., 2016). Essas condições justificam o uso da cobertura vegetal dessas áreas como indicador ambiental (WHITFORD; ENNOS; HANDLEY, 2001).

Indicadores da cobertura vegetal urbana contribuem para avaliar a qualidade ambiental e devem ser considerados nas intervenções urbanas relacionadas à qualidade de vida 
(NUCCI; CAVALHEIRO, 1999). A elaboração e aplicação dessas ferramentas devem ser claras, considerando características climáticas, biogeográficas, geomorfológicas, socioeconômicas, populacionais, históricas e de infraestrutura para garantir seu uso adequado (DA ROCHA; NUCCI, 2018; 2019). Inobstante, informações fornecidas por esses indicadores para avaliação quali-quantitativa das áreas auxiliam na detecção de áreas com degradação/melhoria ecológica (ZHANG et al., 2020) e podem subsidiar a construção de mapas de métricas (SOLTANIFARD; JAFARI, 2019), que orientem um planejamento urbano valorizando os benefícios das áreas verdes para a população urbana (DE OLIVEIRA, 1996; DE CARVALHO, 2001).

Estes índices têm sido utilizados relacionando a área $\left(\mathrm{m}^{2}\right)$ verde urbana e o número de habitantes da cidade (HARDER; RIBEIRO; TAVARES, 2006; BARGOS; MATIAS, 2012; SINGH, 2018; SOLTANIFARD; JAFARI, 2019). García e Guerrero (2006) e De Arruda et al. (2013) avaliaram ainda a área $\left(\mathrm{m}^{2}\right)$ de cobertura vegetal em relação à área impermeabilizada da superfície e à população, respectivamente.

$\mathrm{O}$ presente estudo mediu a cobertura vegetal de Áreas Verdes Públicas (AVP) e avaliou a distribuição dessas áreas na malha urbana da cidade de Governador Valadares, Minas Gerais, Brasil, considerando a população.

Foi testada a hipótese de que estas áreas têm sido mantidas com predominância de cobertura vegetal, o que auxilia na prestação de serviços ecológicos para a população, considerando a premissa de que a cobertura vegetal é proporcional à área das AVPs e estas são maiores em locais mais populosos.

\section{METODOLOGIA}

\section{Área de estudo}

Governador Valadares está localizada no leste de

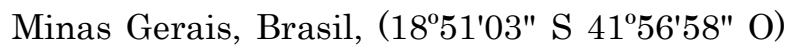
(PMSB, 2015), na região do Vale do Rio Doce. O município possui uma área territorial de 2.342 $\mathrm{km}^{2}$ (IBGE, 2010), com $24,37 \mathrm{~km}^{2}$ de perímetro urbano (PMSB, 2015). A população no ano de 2010 e estimada para 2020 era de 263.689 (96\% urbana) e 281.046 habitantes, respectivamente.
A densidade populacional em 2010 equivalia a $112,58 \mathrm{hab} . / \mathrm{km}^{2}$.

O município está inserido no bioma Mata Atlântica e apresenta florestas do tipo Ombrófila Densa nas partes elevadas e composição Estacional Semidecidual e Decidual nas áreas mais baixas e dissecadas (PMSB, 2015). O clima do município é do tipo AW - tropical subquente e subseco (KÖPPEN, 1948). A temperatura é considerada elevada, chegando a uma média histórica de $26,9{ }^{\circ} \mathrm{C}$ no verão e a $21,5{ }^{\circ} \mathrm{C}$ no inverno. A precipitação e umidade relativa médias anuais equivalem a $1.113,80 \mathrm{~mm}$ e $75 \%$, respectivamente. $\mathrm{O}$ período chuvoso abrange os meses de novembro a janeiro e o seco de julho a setembro (PMSB, 2015).

Governador Valadares está às margens do rio Doce, em uma região que favoreceu sua expansão demográfica e o uso intenso dos recursos naturais, incluindo a exploração mineral e florestal, especialmente no período de 1940 a 1970, gerando problemas ambientais a longo prazo (ESPÍNDOLA, 2015).

\section{Caracterização das $A V P$ s e medição da cobertura vegetal}

A cidade possui 114 lotes cadastrados como AVP pela Prefeitura Municipal de Governador Valadares (PMGV). A instituição das AVPs corresponde à Lei Complementar $\mathrm{n}^{\circ} 178$ do município (GOVERNADOR VALADARES, 2014 ), em que $12 \%$ da gleba pode ser destinado às "áreas reflorestadas com ou sem caminho e trilhas, mantendo-se as características de área verde com existência de vegetação contínua, ampla e livre de edificações".

No levantamento dos dados foram consideradas apenas AVPs do perímetro urbano $\left(24,37 \mathrm{~km}^{2}\right)$ contendo todos os bairros da cidade (Figura 1). Estas AVPs incluem praças e lagoas, sendo a maior parte espaços sem utilização. Elas foram escolhidas, porque são registradas e controladas pela PMGV e, por serem exigidas na abertura de novos loteamentos, viabilizam o monitoramento da cobertura vegetal concomitante à expansão urbana. Das 114 AVPs cadastradas pela PMGV foram delimitadas 78 AVPs e suas respectivas áreas $\left(\mathrm{m}^{2}\right)$, uma vez que diferentes lotes de AVPs conectados foram considerados como uma única AVP. 
Figura 1 - AVPs (em verde) do perímetro urbano de Governador Valadares/MG cadastradas pelo Poder Público Municipal.

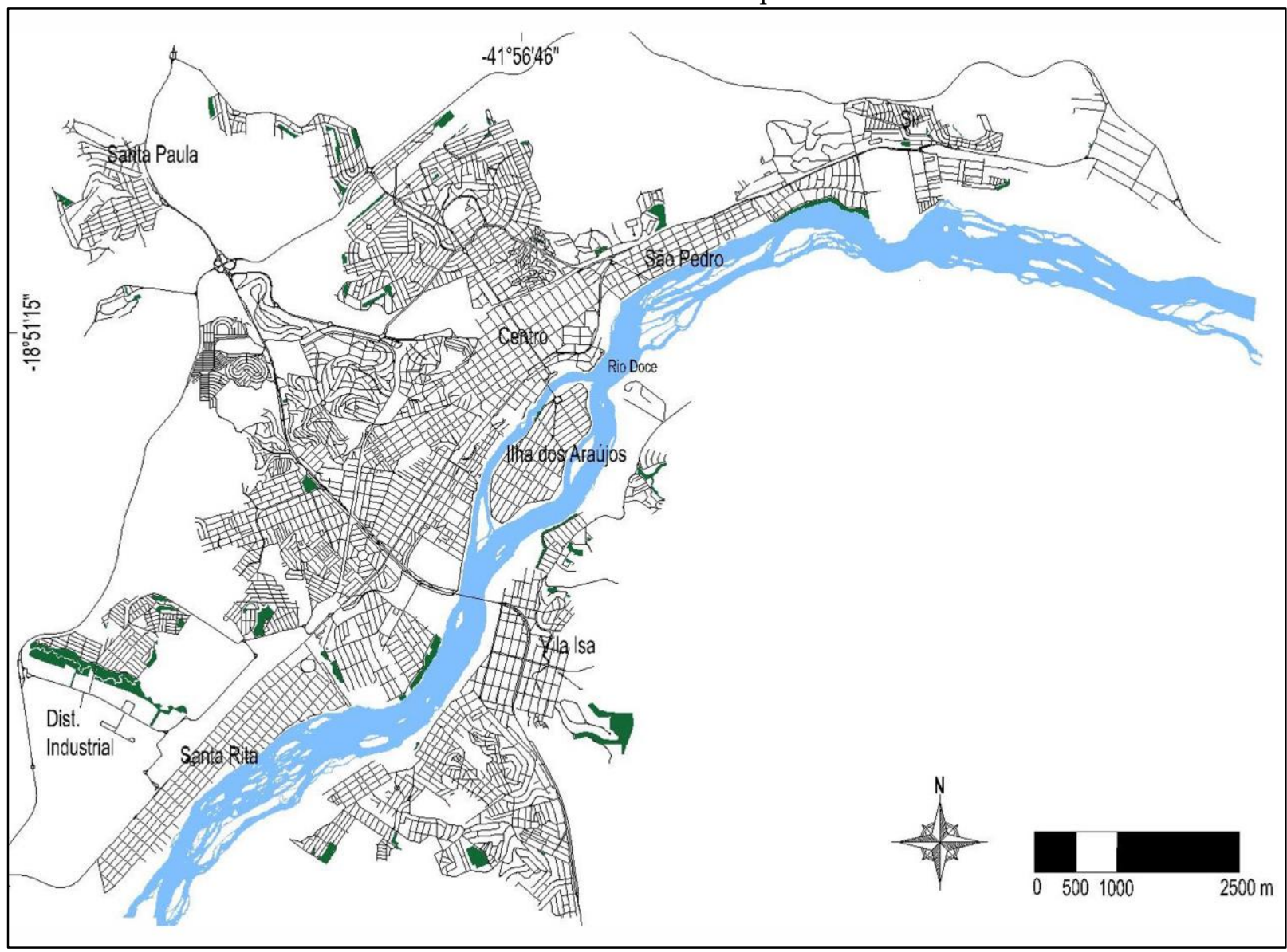

Fonte: PMGV (2017). Adaptado pelos autores, 2018.

Como em muitas AVPs existiam outros componentes além da cobertura vegetal, para melhor distingui-la, em cada AVP foi determinada a área $\left(\mathrm{m}^{2}\right)$ de: Cobertura Vegetal (CV) - projeção do verde em cartas planimétricas (identificado por ortofotografias) (CAVALHEIRO et al., 1999); Área permeável/livre - espaço sem CV; Área construída - edificações presentes no interior das AVPs; e Área de espelho d'água - superfícies de água.

Esse levantamento foi realizado através da formação de polígonos dentro dos limites da AVP, utilizando o software AutoCad ${ }^{(\mathrm{R})} 2018$ (DE CARVALHO, 2001) e uma ortofotografia (GSDGround Sample Distance $10 \mathrm{~cm}-0,1$ pixel/cm) georreferenciada de 2015 fornecida pela PMGV. A partir desse levantamento, foi determinada a proporção de CV em relação à área de cada AVP.

\section{Distribuição das $A V P_{S}$}

A disponibilidade espacial de AVPs e sua relação com as pessoas expressam a ideia de proximidade física, sendo um artifício para estudar a acessibilidade a essas áreas pela população (SINGH, 2018). Foram aplicados dois métodos para avaliar a distribuição das AVPs na malha urbana considerando a população. Inicialmente, as AVPs foram classificadas de acordo com o zoneamento urbano de Governador Valadares (GOVERNADOR VALADARES, 2015): Zona De Adensamento (ZA); Zona Central (ZC); Zona de Habitação de Interesse Social (ZHIS); Zona de Influência das Águas (ZIA); Zona de Interesse Ambiental e Urbano (ZIAU); Zona Industrial e de Grandes Equipamentos (ZIG).

O segundo método contou com o cálculo de índices, com os quais é possível conhecer a oferta de AVP e CV das AVPs na cidade, auxiliando o monitoramento e manutenção dessas áreas (COSTA; FERREIRA, 2011). Foram calculados o Índice de AVP (IAVP) pela Equação 1 e o Índice de CV das AVPs (ICV-AVP) pela Equação 2.

$$
\begin{aligned}
& \operatorname{IAVP}\left(\frac{m^{2}}{\text { hab. }}\right)=\frac{\text { Área total de } \operatorname{AVP}\left(\mathrm{m}^{2}\right)}{\text { Número total de habitantes }} \quad \text { Equação } 1 \\
& I C V-A V P\left(\frac{m^{2}}{\text { hab. }}\right)=\frac{\text { Área total de Cobertura Vegetal da } A V P\left(m^{2}\right)}{\text { Número total de habitantes }} \text { Equação 2 }
\end{aligned}
$$


Para investigar como se deu a distribuição das AVPs ao longo da expansão urbana, foram determinadas as áreas totais $\left(\mathrm{m}^{2}\right)$ de AVP e CV de AVPs para cada bairro existente na cidade. Em seguida foram estabelecidas as faixas etárias dos bairros conforme o Plano Municipal de Saneamento Básico de Governador Valadares (PMSB, 2015), e o número de habitantes e a delimitação de cada bairro foram obtidos junto ao banco de dados do censo de 2010 (IBGE 2010b).

O IAVP e o ICV-AVP também foram computados no âmbito da cidade, considerando todas as AVPs e o número total de habitantes da cidade pela soma da população dos bairros, e em menor escala, ou seja, para cada bairro que constitui a cidade.

\section{Análise de dados}

No intuito de verificar se as AVPs estão sendo mantidas com predominância de cobertura vegetal, componente que conduz à oferta dos serviços ecológicos para a população, analisou-se como a cobertura vegetal está distribuída dentro das AVPs. Portanto, avaliou-se a relação entre a área total de AVP e a sua proporção de CV, através de análises de variância (ANOVA) utilizando o software $R$ (Project for Statistical Computing) (TEAM, 2013).

Para avaliar a distribuição das AVPs considerando a população urbana, a relação da CV com o zoneamento urbano da cidade também foi analisada pela ANOVA. Ainda no sentido de distribuição das AVPs, mas para avaliar o efeito da escala de aplicação dos índices, o IAVP e ICVAVP considerando os dados da cidade como um todo foram comparados com a média dos índices dos bairros. Essa comparação foi realizada para avaliar o possível cenário em que a distribuição das AVPs pode ser mascarada dependendo da escala de aplicação do índice (BARGOS; MATIAS, 2012).
Foi testada também a relação entre os índices calculados anteriormente (IAVP e ICV- AVP), sendo utilizado o mesmo modelo de teste para analisar a relação dos índices com as faixas etárias dos bairros, de modo a avaliar a distribuição de AVPs e CV de AVP ao longo do tempo de expansão urbana da área de estudo. Posteriormente, foi testada a correlação entre a faixa etária de cada bairro com seu número de habitantes para complementar a análise anterior e verificar a relação da existência de AVP e CV com a distribuição da população na cidade.

Finalmente, o IAVP e o ICV-AVP de cada bairro foram classificados, separadamente, em: "Satisfatório" - IAVP/ICV-AVP > $12 \mathrm{~m}^{2} / \mathrm{hab}$., "Não Satisfatório" - IAVP/ICV-AVP $<12 \mathrm{~m}^{2} / \mathrm{hab}$. e "Nulo" - IAVP/ICV-AVP igual a zero. Campinas (2006) adotou este valor para avaliar o desempenho ambiental urbano em relação às áreas verdes e o mesmo número também atende ao intervalo de 10 a $16 \mathrm{~m}^{2} / \mathrm{hab}$., considerado ideal por outros autores (DA SILVA; DOS SANTOS; DE OLIVEIRA, 2016).

\section{RESULTADOS}

As Áreas Verdes Públicas da malha urbana de Governador Valadares estão dispostas na Figura 2 , com suas respectivas proporções de CV. As AVPs apresentam $52,88 \%$ de área permeável/livre, sendo menos da metade do total das áreas estudadas coberto por vegetação (43,34\%) (Tabela 1). As superfícies de água $(1,96 \%)$ e edificações $(1,82 \%)$ situadas no interior das AVPs ocupam a menor parte destas áreas. Mais da metade dos 83 bairros analisados (53\%) não possuem AVP e cinco bairros possuem AVP sem cobertura vegetal. 
Figura 2 - Porcentagem de CV das AVPs do perímetro urbano de Governador Valadares/MG em 2015.

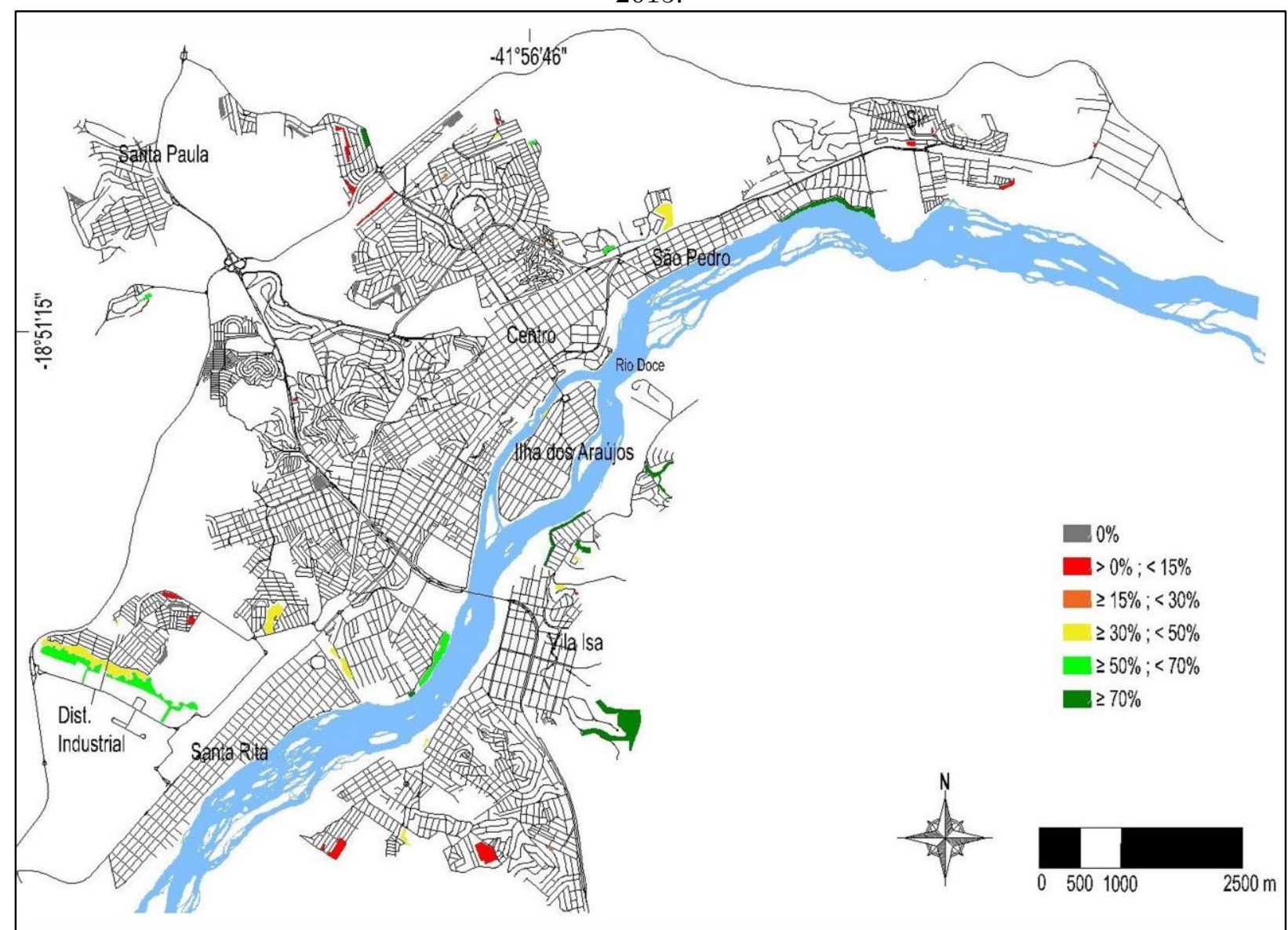

Org: Autores, 2018.

Tabela 1. Caracterização física das AVPs $\left(\mathrm{m}^{2}\right)$ de Governador Valadares/MG.

\begin{tabular}{lll}
\hline Área total de AVP & $1.159 .265,31(\mathrm{~m} 2)$ & $\mathbf{1 0 0 \%}$ \\
\hline Área Permeável & $613.077,15$ & $52,88 \%$ \\
Área de Cobertura Vegetal & $502.428,30$ & $43,34 \%$ \\
Área com Espelho d'água & $22.686,15$ & $1,96 \%$ \\
Área construída & $21.073,71$ & $1,82 \%$ \\
\hline
\end{tabular}

Org: Autores, 2018.

Observou-se uma correlação positiva entre a Proporção de CV e a metragem quadrada das $\operatorname{AVPs}\left(\mathrm{F}_{(1,76)}=4,3665 ; \mathrm{p}=0,04\right)$, indicando que, com o aumento da área de AVP a proporção de CV também se eleva (Figura 3). 
Figura 3 - Relação entre a CV e a área das AVPs de Governador Valadares/MG.

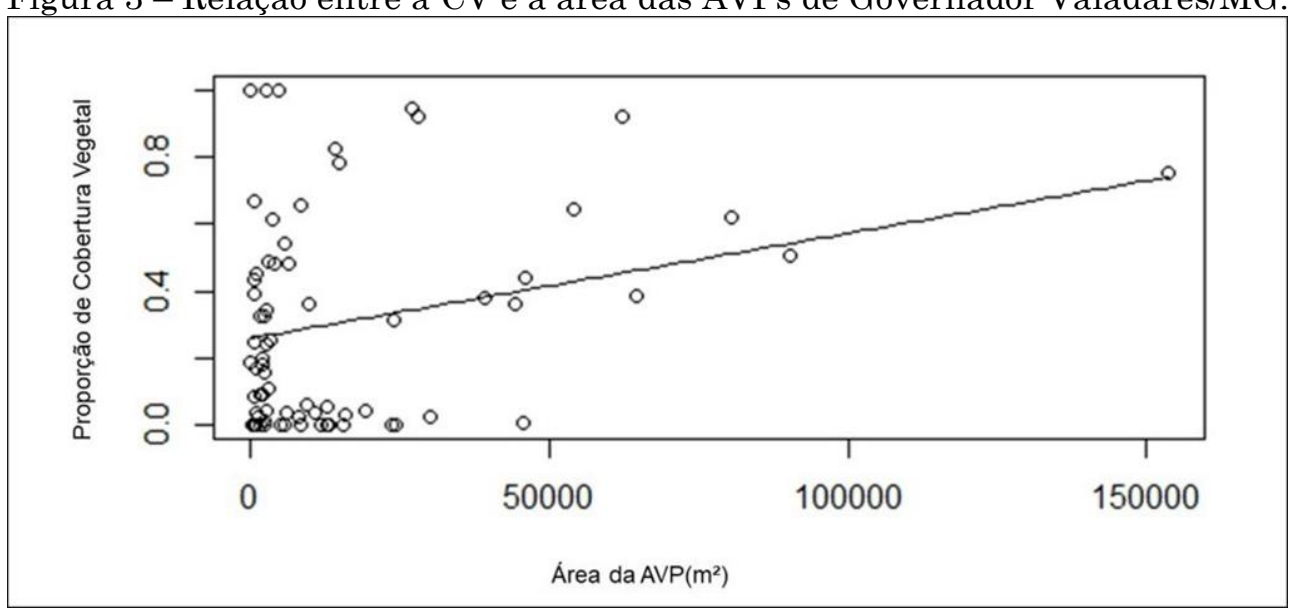

Org: Autores, 2018.

Quando analisada a relação da CV das AVPs com os setores do zoneamento urbano da cidade, foi constatado que há diferenças significativas entre as áreas $\left(\mathrm{F}_{(9,68)}=5,061 ; \mathrm{p}=3,155 \mathrm{e}-05\right)$, semelhante aos resultados de Soltanifard e Jafari (2019). A maioria das zonas apresentou AVPs com baixos valores de CV (Figura 4), destacando-se a ZA, constituída por bairros populosos com tendência à expansão urbana contínua, apresentando baixos valores de CV. A ZIAU além de apresentar uma maior variação dos dados, obteve uma baixa média nos valores de CV. Por outro lado, a ZIG se diferencia pelos maiores valores de CV.

Figura 4 - Distribuição da CV das AVPs nos setores de zoneamento urbano de Governador Valadares/MG. ZA: Zona de Adensamento; ZC: Zona Central; ZHIS: Zona de Habitação de Interesse Social; ZIA: Zona de Influência das Águas; ZIAU: Zona de Interesse Ambiental e Urbano; ZIG: Zona Industrial e de Grandes Equipamentos.

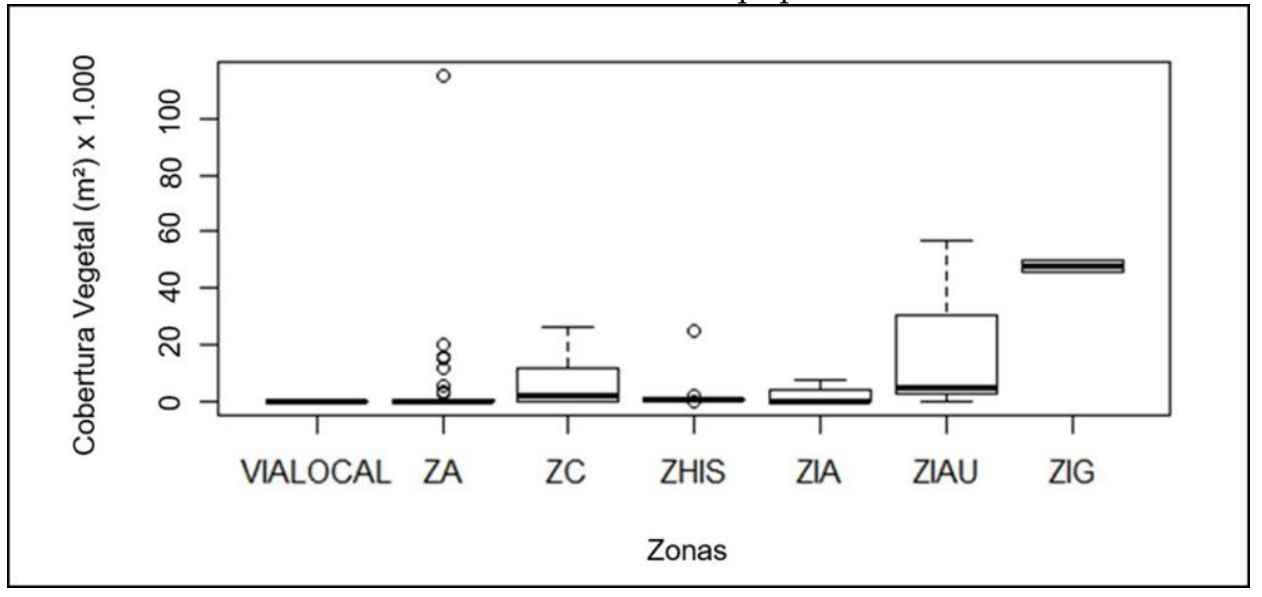

Org: Autores, 2018.

O IAVP e o ICV-AVP calculado para cidade e a média dos índices dos bairros apresentaram diferença significativa, sendo a média sempre maior (Tabela 2). Os índices para a cidade podem ser considerados baixos, comparados ao de 21,7 $\mathrm{m}^{2} /$ hab. registrado em uma cidade na Índia com população aproximada (285 mil hab.) (SINGH, 2018).

Tabela 2. IAVP e ICV-AVP de Governador Valadares/MG.

\begin{tabular}{lcc}
\hline & Cidade & Média dos bairros \\
\hline $\operatorname{IAVP}\left(\mathrm{m}^{2} /\right.$ hab. $)$ & 4,77 & 158,27 \\
ICV-AVP $\left(\mathrm{m}^{2} / \mathrm{hab}.\right)$ & 2,07 & 36,52 \\
\hline
\end{tabular}

Org: Autores, 2018. 
Relacionando o IAVP e o ICV-AVP dos bairros, notou-se a correlação positiva entre as duas variáveis $\quad\left(\mathrm{F}_{(1,71)}=5,223 ; \quad \mathrm{p}=0,02528\right)$. Portanto, para as análises seguintes optou-se somente pelo ICV-AVP, que é inerente à CV das AVPs, ou seja, à prestação de serviços ecológicos pela vegetação urbana. Assim, verificou-se uma redução do ICV-AVP com o aumento da faixa etária dos bairros (Figura 5a) $\quad\left(\mathrm{F}_{(1,71)}=4,2523\right.$; $\mathrm{p}=0,04286$. Uma análise que complementa os resultados anteriores consiste na relação entre o número de habitantes residentes nos bairros e a faixa etária destes $(\mathrm{F}(1,71)=39,755 ; \mathrm{p}=2,148 \mathrm{e}-8)$, sendo apurado que a população é maior quando se trata de bairros mais antigos (Figura 5b). Então, os resultados da Figura 5 indicam que a CV das AVPs é menor em bairros mais antigos que, por sua vez, são mais populosos.

Figura 5 - ICV-AVP (a) e população residente (b) em bairros com diferentes idades de Governador Valadares/MG.

(a)

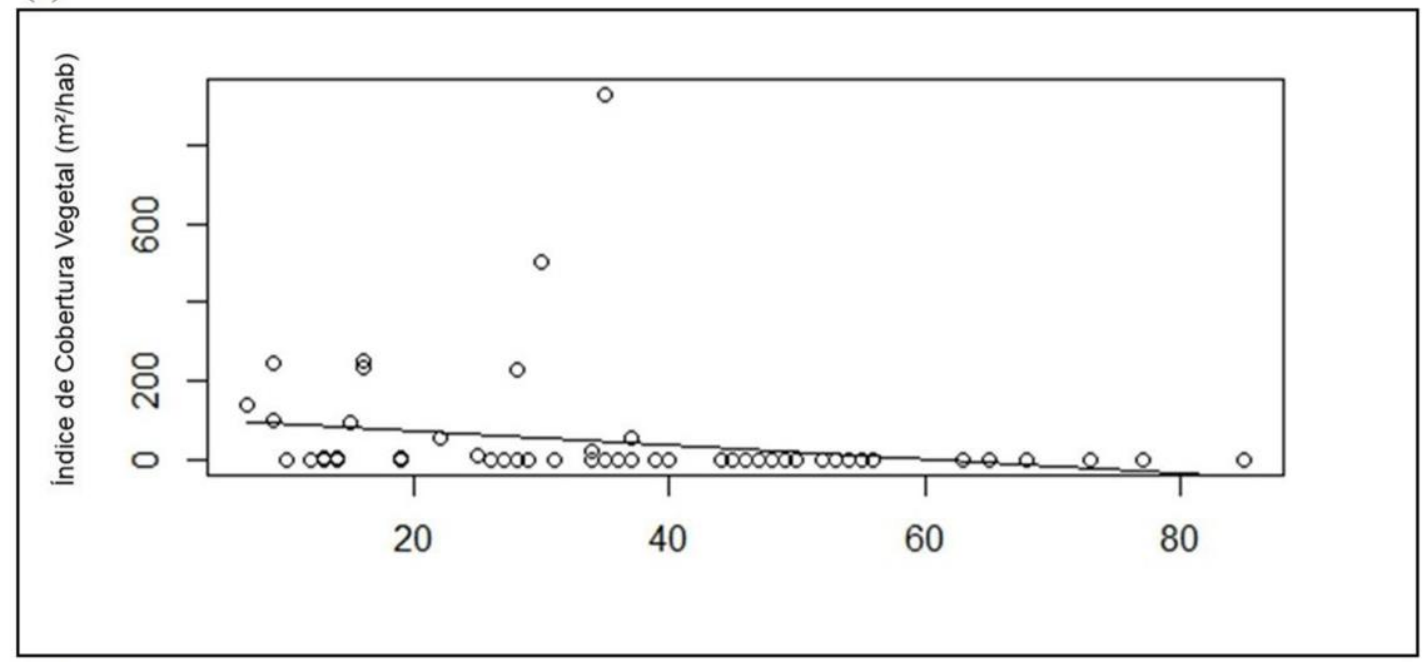

(b)

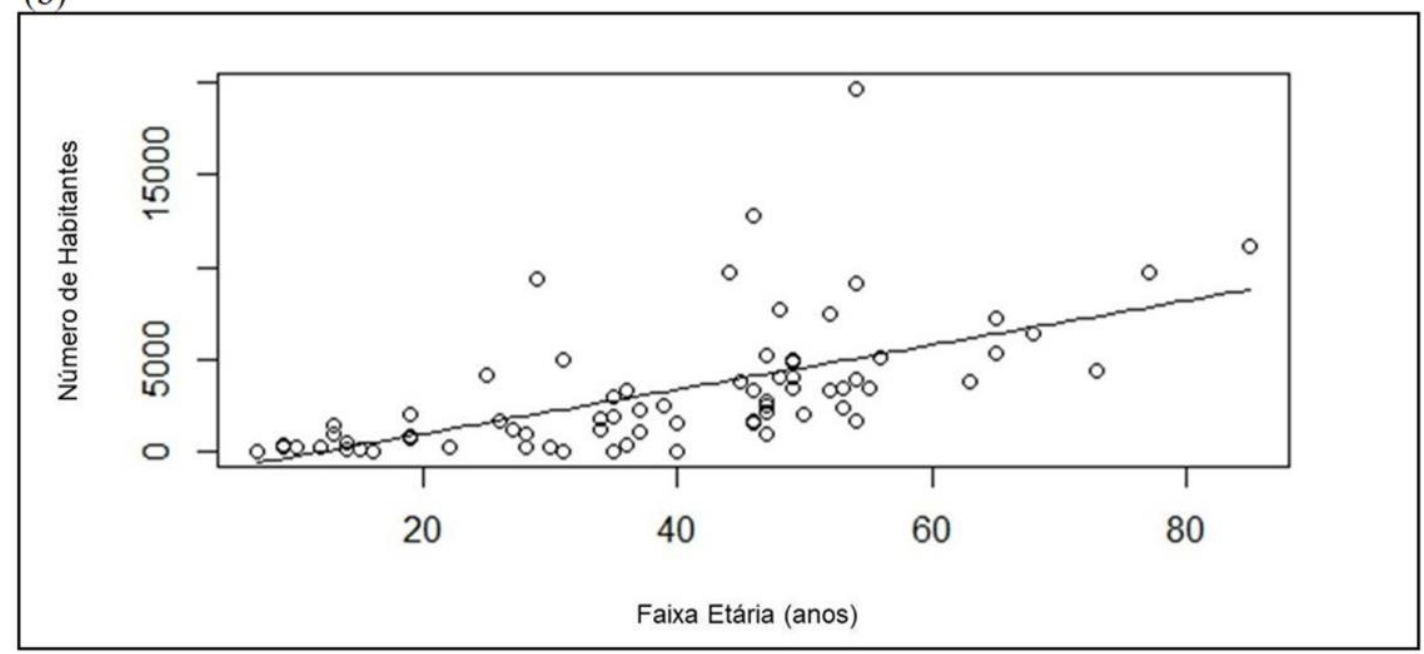

Org: Autores, 2018.

A partir da classificação do IAVP e ICV-AVP de cada bairro pelo parâmetro de $12 \mathrm{~m}^{2} / \mathrm{hab}$. (CAMPINAS, 2006), notou-se que Governador Valadares é composta em sua maioria por bairros com índices nulos, indicando ausência de
AVP (Figura 6a) e de CV nas AVPs (Figura 6b). É evidente ainda que o número de índices Satisfatórios diminui de $25 \%$ para $16 \%$ nos bairros da cidade, do IAVP para o ICV-AVP, respectivamente. 
Figura 6 - Classificação do IAVP (a) e ICV-AVP (b) para os bairros de Governador Valadares/MG. Não Satisfatório: menor que $12 \mathrm{~m}^{2} /$ hab.; Satisfatório: maior que $12 \mathrm{~m}^{2} / \mathrm{hab}$.

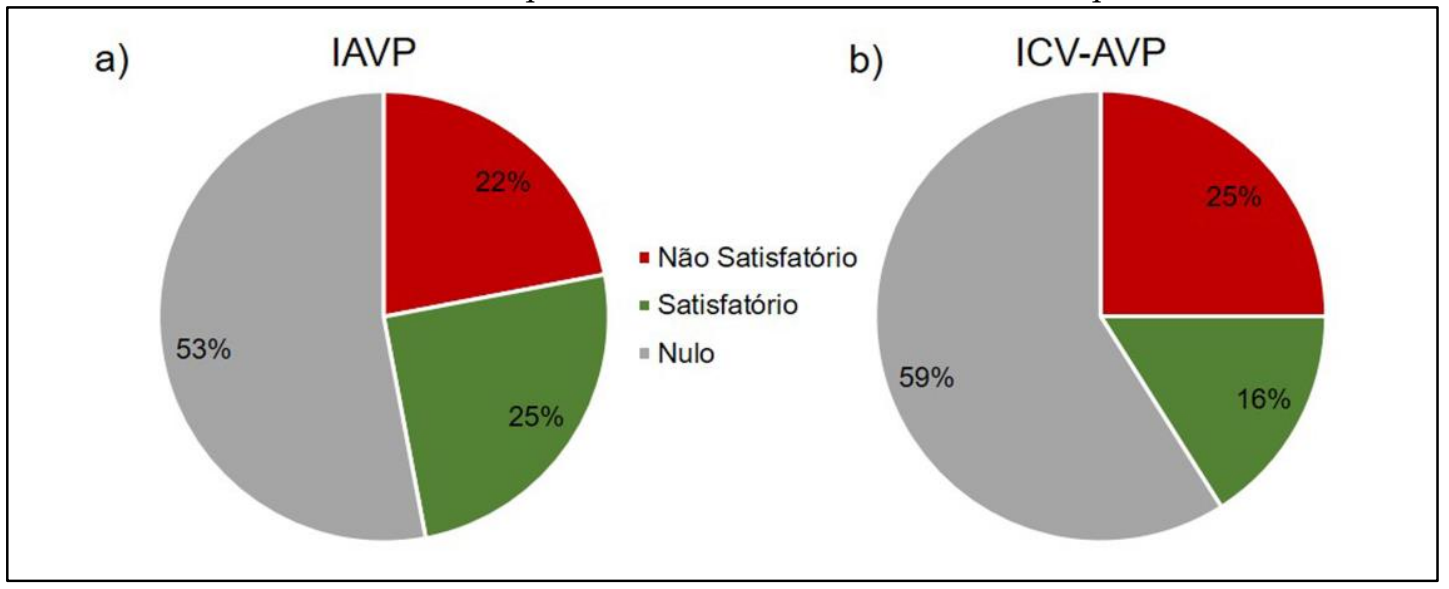

Org: Autores, 2018.

\section{DISCUSSÃO}

Os valores absolutos apresentados na Tabela 1 evidenciam que menos de $5 \%$ da área urbana de Governador Valadares é formado por AVPs, valor inferior ao encontrado por outro estudo $(9,1 \%)$ em cidade com número aproximado de habitantes (SINGH, 2018). Somando Área Permeável e Cobertura Vegetal ultrapassa-se $70 \%$ da área total de AVPs, limite mínimo recomendado (GUZZO; CARNEIRO; OLIVEIRA JÚNIOR, 2006). Esse resultado aparenta ser favorável, entretanto, se tratando apenas da CV, o valor não representa sequer $50 \%$ do valor total da área de AVPs.

Inobstante, a Figura 3 revela que a distribuição da CV nas AVPs não é uniforme, sendo que, independente da área da AVP, a proporção de CV deveria ser constante e, preferencialmente, superior a $70 \%$ da área. Como nenhuma das duas situações acontece na maioria das AVPs, esses resultados (Tabela 1 e Figura 3) são fortes indícios de que a CV não é priorizada nas AVPs avaliadas. Essas circunstâncias são contrárias à previsão da legislação (BRASIL, 2012; GOVERNADOR VALADARES, 2014) que define as AVPs como áreas com predomínio de cobertura vegetal, o que garante seus serviços ecológicos (NUCCI; CAVALHEIRO, 1999; EDWARDS, 2008).

A Figura 2 ilustra esse cenário e pode ser considerado uma ferramenta métrica para analisar a heterogeneidade espacial urbana em relação às $\mathrm{AVPs}$, constituindo um status de espaços verdes (SOLTANIFARD; JAFARI, 2019). Essa informação é valiosa no processo de tomada de decisões e ajuda a certificar que as ações estejam sendo realizadas para melhorar a
CV urbana. Na Figura 2 é possível observar a dominância de AVPs com CV inferior a 70\% da área da AVP, sendo que apenas 12 AVPs, das 78 analisadas, apresentaram $\mathrm{CV}$ de valor igual ou maior que esse. Dessa parcela, cinco estão situadas próximas ao limite do perímetro urbano e consequentemente afastadas de zonas mais habitadas. A Figura 2 corrobora resultados recentes (SINGH, 2018), em que é visível a distribuição desigual das AVPs na malha urbana, aumentando a heterogeneidade espacial e influenciando negativamente a qualidade ecológica das AVPs (SOLTANIFARD; JAFARI, 2019). Enquanto que, a localização de AVPs em pontos distantes da população representa limitações dos efeitos positivos da vegetação de AVPs sobre a população urbana.

Os resultados da análise considerando o zoneamento urbano em primeiro momento são animadores pela maior parcela das AVPs estar localizada na ZA (regiões urbanizadas e populosas), promovendo, portanto, ligação entre as pessoas e os benefícios das AVPs. Contudo, estas AVPs apresentam baixos valores de CV (Figura 4), com média quase nula. Contraditoriamente, a ZIG foi a zona que apresentou os maiores valores de CV nas AVPs e as áreas dessas AVPs representam cerca de 15\% do total existente na cidade. Os valores de CV para a ZIG são altos para uma zona pouco habitada, quando se pensa nos benefícios das AVPs para a população urbana. Inobstante, como ainda existem chances de crescimento do setor industrial na cidade (PMSB, 2015), essas AVPs estão susceptíveis à degradação mediante à possibilidade de chegada de novas industrias (HARDER, 2002; SINGH, 2018). Esses resultados reforçam a importância de garantir altas taxas de CV nas AVPs e ainda alertam que 
é preciso não apenas conservar AVPs em zonas industriais, como também prezar pela existência das mesmas em zonas densamente habitadas, contribuindo para a melhoria na qualidade da vida urbana, particularmente para a saúde física e mental das pessoas (DE OLIVEIRA, 1996; NUCCI; CAVALHEIRO, 1999; DE CARVALHO, 2001; LOBODA; DE ANGELIS, 2005).

Ainda a respeito de zonas pouco habitadas, esperava-se que as AVPs situadas na ZIAU apresentassem altos valores de $\mathrm{CV}$, o que não acontece (Figura 4). Além disso, do total de AVPs existentes na cidade, uma pequena parte $(9 \%)$ está na ZIAU e $57 \%$ desta parcela é considerada como Área de Preservação Permanente (APP), por se localizar na mata ciliar do rio Doce. Essas APPs que foram cadastradas como AVPs estão ainda entre as 12 AVPs que possuem mais de $70 \%$ de sua área coberto por vegetação. Esse cenário denota o merecimento de políticas ambientais urbanas para a recuperação, manutenção, monitoramento e fiscalização dessas áreas, de modo a garantir seu valor ecológico, histórico, cultural, paisagístico e turístico (MMA, 2020).

A escala de aplicação dos índices (IAVP e ICVAVP), por sua vez, remete ao sistema políticoadministrativo de divisão territorial das cidades no Brasil - bairros, zoneamento - que deve direcionar políticas públicas para as AVPs. Bairros recém implantados apresentaram maiores valores de ICV-AVP (Figura 5a). Contudo, a localização desses bairros em áreas de interface urbana/rural com características de espaços verdes pode ser uma explicação para os altos índices (SOLTANIFARD; JAFARI, 2019). Também é importante considerar que muitos desses bairros ainda não estão plenamente urbanizados e que com o aumento da população o índice deve sofrer alteração se nenhuma medida for realizada para conservação e/ou aumento proporcional de AVPs com CV.

Atenção também para os bairros mais antigos que não possuem AVPs (Figura 5a), os quais apresentam maior população (Figura 5b) e estão localizados próximos a região central da cidade. O mesmo foi observado por De Paula e Ferreira (2017), evidenciando que centros e regiões mais antigas de cidades comumente sofrem com falta de planos para o desenvolvimento de áreas verdes (SINGH, 2018). Logo, a população de tais bairros pode não ser beneficiada pelos serviços ambientais prestados pelas AVPs, o que chama mais atenção diante do enfrentamento da pandemia de COVID-19. As AVPs podem auxiliar a prevenir, de forma segura, os efeitos negativos sobre a saúde física e psicológica da população durante o distanciamento social
(GUADAGNIN, 2020; SANTOS; 2020). Por isso, outras alternativas como a arborização viária (SALVI et al., 2011) e revitalização de praças (BARROS; VIRGILIO, 2003) podem contribuir. Essas medidas ajudariam ainda a evitar a fragmentação de espaços verdes e criar maiores conexões ecológicas na cidade, aumentando a estabilidade ecológica e melhorando a qualidade do ambiente urbano (SOLTANIFARD; JAFARI, 2019).

Diante da classificação recomendada por Campinas (2006) - $12 \mathrm{~m}^{2}$ de AVP para cada habitante - os resultados obtidos nesse estudo revelam que apenas um quarto dos bairros estudados atende à recomendação para o IAVP, sendo que, tanto as áreas verdes (Figura 6a) quanto a cobertura vegetal (Figura 6b), sequer existem em mais da metade dos locais estudados. Quando se adota a recomendação da Sociedade Brasileira de Arborização Urbana - $15 \mathrm{~m}^{2} / \mathrm{hab}$. (DE ARRUDA et al., 2013; LUCON; DO PRADO FILHO; SOBREIRA, 2013), a situação é mais preocupante. Além disso, pela diminuição dos registros satisfatórios de $25 \%$ para $16 \%$, do IAVP para o ICV-AVP, respectivamente, se nota que parte das AVPs apesar de possuírem tamanho de acordo com o recomendado, não apresentam cobertura vegetal, sendo necessário o reflorestamento dessas áreas.

Recomenda-se o uso do ICV-AVP para a avaliação das AVPs, conforme já sugerido (CAVALHEIRO; NUCCI, 1998; NUCCI, 2008). Este índice pode auxiliar na visualização de mudanças nos serviços ecológicos, orientando um planejamento urbano para áreas com degradação/melhoria ecológica (ZHANG et al., 2020). O ICV-AVP pode ainda complementar outros índices que avaliam efeitos da vegetação sobre o mercado imobiliário (JIAO et al., 2017) ou analisam as condições físicas da vegetação e do solo (DHAWALE; PAUL, 2018).

Os resultados apresentados são inerentes à $\mathrm{CV}$ existente em AVPs e desconsideram a CV em outros locais da cidade, que também deveria ser registrada pela PMGV, como a arborização viária, parques e áreas de proteção ambiental. De qualquer forma, o retrato da CV das AVPs, áreas controladas pela prefeitura, auxilia a monitorar o atendimento à legislação por novos loteamentos e, portanto, a relação entre a CV e o crescimento da cidade. Inobstante, o acompanhamento de todas as áreas verdes urbanas contribuiria ainda mais para a conservação da cobertura vegetal na cidade. 


\section{CONCLUSÃO}

As AVPs somam cerca de $1,15 \mathrm{~km}^{2}$ da área de estudo, embora representem menos de $5 \%$ do total da malha urbana. Menos da metade da área das AVPs é coberto por vegetação e a CV existente é inconstante, ocupando, na maior parte das unidades, menos de $70 \%$ de sua área. É necessário criar espaços verdes em regiões mais antigas e sem AVPs e conservar e reflorestar as AVPs com CV satisfatória e insatisfatória, respectivamente.

A legislação para instituição de AVPs certamente auxilia na reversão do histórico de degradação ambiental da expansão urbana, apesar de não se mostrar suficientemente clara sobre aspectos de identificação/implementação dessas áreas. Não obstante, o status de distribuição desigual de AVPs e sua CV aponta que essas áreas não têm sido mantidas prioritariamente com predomínio de vegetação. As AVPs existem provável/exclusivamente em resposta à legislação/fiscalização mais incidente sobre a recente urbanização.

Os índices - IAVP e ICV-AVP - e o mapa de distribuição da CV das AVPs (Figura 2) constituem ferramentas adequadas e de baixo custo que viabilizam uma análise da cobertura vegetal urbana. A partir de uma ortofotografia de 0,1 pixell $\mathrm{cm}$, foi gerada informação de qualidade para o planejamento urbano, visando os serviços ambientais que a vegetação oferece à população. Recomenda-se estudos relacionando estas ferramentas com a distância física e fatores econômicos da população, bem como com uma caracterização específica da vegetação existente e um monitoramento a médio/longo prazo de AVPs.

\section{AGRADECIMENTOS}

Agradecemos ao Núcleo de Ciências e Tecnologia e ao Programa de Pós-Graduação Stricto Sensu em Gestão Integrada do Território da Universidade Vale do Rio Doce pelo suporte à presente pesquisa. Também agradecemos à Secretaria de Planejamento da Prefeitura Municipal de Governador Valadares por fornecer os dados usados neste estudo, assim como à CAPES, FAPEMIG, CNPq e ANA.

\section{REFERÊNCIAS}

BARGOS, D. C.; MATIAS, L. F. Mapeamento e
Análise de Áreas Verdes Urbanas em Paulínia (SP): estudo com a aplicação de geotecnologias. Sociedade e Natureza, Uberlândia, ano 24, n.1, p. 143-156, 2012. https://doi.org/10.1590/S198245132012000100012

BARROS, M. V. F.; VIRGILIO, H. Praças: espaços verdes na cidade de Londrina. Geografia: Londrina, v. 12, n. 1, p. 533-544, 2003. http://dx.doi.org/10.5433/24471747.2003v12n1p533

BOLDRIN, K. V. F.; GARCIA, C. S. G.; PAIVA, P. D. O.; DE CARVALHO, L. M. Quantitative inventory and analysis of the green areas in Lavras-MG and index evolution. Ornamental Horticulture, v. 22, n. 2, p.138-142, 2016. https://doi.org/10.14295/oh.v22i2.769

BONZI, R. Paisagem como infraestrutura. In: PELLEGRINO, P.; MOURA, N. (Org). Estrategias para uma infraestrutura verde. 1. ed. Rio de Janeiro: Manole, 2017. p. 1-24.

BRASIL. Lei 12.651, de 25 de maio de 2012. Dispõe sobre a proteção da vegetação nativa; altera as Leis nos 6.938, de 31 de agosto de 1981, 9.393, de 19 de dezembro de 1996, e 11.428, de 22 de dezembro de 2006; revoga as Leis nos 4.771, de 15 de setembro de 1965, e 7.754, de 14 de abril de 1989, e a Medida Provisória no 2.166-67, de 24 de agosto de 2001; e dá outras providências. Diário Oficial da União, Brasília, 2012. Disponível em: $<$ http://www.planalto.gov.br/ccivil_03/_Ato20112014/2012/Lei/L12651.htm>. Acesso em: 20 mai. 2018.

CAMPINAS. Prefeitura Municipal. Lei No 12.585 de 28 de junho de 2006. Dispõe sobre a instituição de metas e Índices de Desempenho Ambiental no Município de Campinas e dá outras providências. Campinas: Coordenadoria Setorial de Documentação - Biblioteca Jurídica; 2006. Disponível em: <https://cmcampinas.jusbrasil.com.br/legislacao/317875/lei12585-06>. Acesso em: 20 mai. 2018.

CAVALHEIRO, F.; NUCCI, J. C. Espaços Livres e Qualidade de Vida Urbana. Paisagem Ambiente: São Paulo, n. 11, p. 277-288, 1998. https://doi.org/10.11606/issn.2359-

5361.v0i11p277-288

CAVALHEIRO, F.; NUCCI, J. C.; GUZZO, P.; ROCHA, Y. T. Proposição de Terminologia para o Verde Urbano. Boletim Informativo da Sociedade Brasileira de Arborização Urbana. Rio de Janeiro: Ano VII, n. 3, 1999. Disponível em: <https://tgpusp.files.wordpress.com/2018/05/cava lheiro-et-al-1999.pdf>. Acesso em 17 nov. 2020.

COSTA, R. G.; FERREIRA, C. C. M. Estudo das Áreas Verdes em Juiz de Fora, MG. Revista de Geografia - PPGEO - v. 1, no 1, p. 1-10, 2011.

DA ROCHA, M. F.; NUCCI, J. C. Cobertura Vegetal na Região Central das Capitais Brasileiras. GEOgraphia, v. 21, n.45, p. 70-85, 2019.

https://doi.org/10.22409/GEOgraphia2019.v21i45 
.a14352

DA ROCHA, M. F.; NUCCI, J. C. Índices de vegetação e competitividade entre cidades. GEOUSP Espaço e Tempo (Online), v. 22, n.3, p. 641-655, 2018. https://doi.org/10.11606/issn.21790892.geousp.2018.133554

DA SILVA, A. D. P.; DOS SANTOS, A. F.; DE OLIVEIRA, L. M. Índices de Área Verde e Cobertura Vegetal das Praças Públicas da Cidade de Gurupi, TO. Floresta, Curitiba, PR, v. 46, n. 3, p. 353-361, 2016. http://dx.doi.org/10.5380/rf.v46i3.40052

DE ARRUDA, L. E. V.; SILVEIRA, P. R. S; VALE, H. S. M.; DA SILVA, P. C. M. Índice de área verde e de cobertura vegetal no perímetro urbano central do município de Mossoró, RN. Revista Verde de Agroecologia e Desenvolvimento Sustentável, Mossoró, RN, v. 8, n. 2, p. 13-17, 2013.

DE CARVALHO, L. M. Áreas verdes da cidade de Lavras/MG: caracterização, usos e necessidades. 2001. 115 f. Dissertação (Mestrado em Agronomia) - Universidade Federal de Lavras, Lavras/MG, 2001.

DE OLIVEIRA, C. H. Planejamento ambiental na cidade de São Carlos (SP) com ênfase nas áreas públicas e áreas verdes: diagnóstico e propostas. 1996. 181 f. Dissertação (Mestrado em Ecologia) Universidade Federal de São Carlos, São Carlos/SP, 1996.

DE PAULA, I. F. M.; FERREIRA, C. C. M. Avaliação e mapeamento da cobertura vegetal da região central da cidade de Juiz de Fora - MG. Ra'ega, Curitiba, vol. 39, p. 146-166, 2017. http://dx.doi.org/10.5380/raega.v39i0.43374

DHAWALE, R.; PAUL, S. K. A comparative analysis of drought indices on vegetation through remote sensing for Latur region of India. International Archives of the Photogrammetry, Remote Sensing and Spatial Information Sciences, vol. XLII-5, 403-407, 2018. https://doi.org/10.5194/isprs-archives-XLII-5403-2018

EDWARDS, B. O guia básico para a sustentabilidade. Brasil: GG Brasil, 2008.

ESPINDOLA, H. S. Vale do Rio Doce: Fronteira, industrialização e colapso socioambiental. Fronteiras: Journal of Social, Technological and Environmental Science, v. 4, p. 160, 2015. https://doi.org/10.21664/2238-

8869.2015v4i1.p160-206

FARR, D. Urbanismo sustentável - desenho urbano com a natureza. 1.ed. Porto Alegre: Bookman, 2013.

FEIBER, S. D. Áreas verdes urbanas imagem e uso - o caso do passeio público de Curitiba-PR. Ra'ega, Curitiba, n. 8, p. 93-105, 2004. https://doi.org/10.5380/raega.v8i0.3385

GARCÍA, S.; GUERRERO, M. Indicadores de sustentabilidade ambiental en la gestión de espacios verdes. Parque Urbano Monte Calvario,
Tandil, Argentina. Revista de geografia Norte Grande, Santiago, n. 35, p. 45-57, 2006. https://doi.org/10.4067/S0718-

34022006000100004

GOVERNADOR VALADARES (Município). Lei Complementar $\mathrm{n}^{\circ}$ 178, de 04 de setembro de 2014 . Dispões sobre o parcelamento do solo para fins urbanos no município de Governador Valadares e dá outras providências. Prefeitura Municipal de Governador Valadares, Minas Gerais, 2014. $36 \mathrm{f}$.

GOVERNADOR VALADARES (Município). Lei Complementar $\mathrm{n}^{\circ}$ 201, de 19 de outubro de 2015. Dispões sobre o uso e a ocupação do solo no município de Governador Valadares e dá outras providências. Prefeitura Municipal de Governador Valadares, Minas Gerais, 2015. $37 \mathrm{f}$. GUADAGNIN, D. L. 2020. Áreas verdes e o convívio social seguro durante a pandemia. Jornal da Universidade. URGS. Disponível em: <https://www.ufrgs.br/jornal/posicionamentoeditorial/>. Acesso em: 08 set. 2020.

GUZZO, P.; CARNEIRO, R. M. A.; OLIVEIRA JÚNIOR, H. Cadastro Municipal de Espaços Livres Urbanos de Ribeirão Preto (SP): acesso público, índices e base para novos instrumentos e mecanismos de gestão. Revista da Sociedade Brasileira de Arborização Urbana, v. 1, n. 1, p. 1930, 2006. https://doi.org/10.5380/revsbau.v1i1.66437

HARDER, I. C. F. Inventário quali-quantitativo da arborização e infraestrutura das praças da cidade de Vinhedo em São Paulo. 2002. 140 f. Dissertação (Mestrado em Agronomia) Universidade de São Paulo, São Paulo/SP, 2002.

HARDER, I. C. F.; RIBEIRO, R. C. S.; TAVARES, A. R. Índices de área verde e cobertura vegetal para as praças do Municipio de Vinhedo, SP. Revista Árvore. vol. 30, n. 2. p. 277 - 282. 2006. https://doi.org/10.1590/S010067622006000200015

IBGE - Instituto Brasileiro de Geografia e Estatística. IBGE/Cidades 2010. Disponível em: $<$ https://cidades.ibge.gov.br/brasil/mg/governadorvaladares/panorama>. Acesso em: 28 fev. 2018.

IBGE - Instituto Brasileiro de Geografia e Estatística. Mapas. Bases e referências - bases cartográficas - malhas digitais. 2010b. Disponível em: <https://mapas.ibge.gov.br/bases-ereferenciais/bases-cartograficas/malhas-

digitais>. Acesso em: 20 mai. 2018.

JIAO, L.; XU, G.; JIN, J.; DONG, T.; LIU, J.; WU, Y.; ZHANG, B. Remotely sensed urban environmental indices and their economic implications. Habitat International, v. 67, p. 2232 , 2017. http://dx.doi.org/10.1016/j.habitatint.2017.06.012 KÖPPEN, W. Climatologia con un estudio de los climas de la tierra. Mexico: Fundo de Cultura Economica, 1948. 478 p.

LOBODA, C. R.; DE ANGELIS, B. L. D. Áreas verdes públicas urbanas: conceitos, usos e 
funções. Ambiência, v. 1, n. 1, p. 125-139, 2005. Disponível em: $<$ https://revistas.unicentro.br/index.php/ambienc ia/article/view/157/185>. Acesso em: 23 fev. 2018.

LUCON, T. N.; DO PRADO FILHO, J. F.; SOBREIRA, F. G. Índice e percentual de áreas verdes para o perímetro urbano de Ouro Preto, MG. Revista da Sociedade Brasileira de Arborização Urbana, Piracicaba, SP, v. 8, n. 3, p. 63-78, 2013.

https://doi.org/10.5380/revsbau.v8i3.66430

MMA - Ministério do Meio Ambiente. Cidades Sustentáveis - Áreas Verdes Urbanas - Áreas de Preservação Permanente Urbanas. Brasília, MMA, 2020. Disponível em: $<$ https://www.mma.gov.br/cidades-

sustentaveis/areas-verdes-

urbanas/\%C3\%A1reas-de-

prote $\% \mathrm{C} 3 \% \mathrm{~A} 7 \% \mathrm{C} 3 \% \mathrm{~A} 30-$ permanente.html>.

Acesso em 17 nov.2020.

NUCCI, J. C. Qualidade Ambiental e Adensamento Urbano: um estudo de ecologia e planejamento da paisagem aplicado ao distrito de Santa Cecília (MSP). João Carlos Nucci, Curitiba, 2 ed., 2008. 150p. Disponível em: <https://tgpusp.files.wordpress.com/2018/05/qual idade-ambiental-e-adensamento-urbano-nucci2008.pdf>. Acesso em 17 nov. 2020.

NUCCI, J. C.; CAVALHEIRO, F. Cobertura vegetal em áreas urbanas: conceito e método. GEOUSP, v. $3, \quad$ n. $2, \quad$ p. $29-36, \quad 1999$. https://doi.org/10.11606/issn.21790892.geousp.1999.123361

PMGV - Prefeitura Municipal de Governador Valadares. Secretaria de Planejamento. Mapa de Áreas Verdes Públicas. Governador Valadares: PMGV, 2017.

PMSB - Plano Municipal de Saneamento Básico. Produto 2 - Diagnóstico da Situação da Prestação dos Serviços de Saneamento Básico: Caracterização Geral do Município. Prefeitura Municipal de Governador Valadares, Governador Valadares, 2015. Disponível em: <https://www.valadares.mg.gov.br/abrir_arquivo. aspx/Diagnostico_caracterizacao_geral_do_munic ipio?cdLocal=2\&arquivo=\%7B64DC8181-E112B640-6A1B-ACE07ED43A42\%7D.pdf>. Acesso em 20 mar. 2018.

SALVI, L. T.; HARDT, L. P. A.; ROVEDDER, C. E.; FONTANA, C. S. Arborização ao longo de ruas Túneis verdes - em Porto Alegre, RS, BRASIL: Avaliação quantitativa e qualitativa. Revista Árvore, Viçosa-MG, v.35, n.2, p. 233-243, 2011. https://doi.org/10.1590/S010067622011000200008
SANTOS, B. S. A cruel pedagogia do vírus. Almedina. 2020. 23pp.

SINGH, K. K. Urban green space availability in Bathinda City, India. Environ. Monit. Assess, 190: 671, 2018. https://doi.org/10.1007/s10661018-7053-0

SOLTANIFARD, H.; JAFARI, E. A conceptual framework to assess ecological quality of urban green space: a case study in Mashhad city, Iran. Environ. Dev. Sustain., vol. 21, p. 1781-1808, 2019. https://doi.org/10.1007/s10668-018-0103-5

TEAM, R. Core. R: A language and environment for statistical computing. $\mathrm{R}$ Foundation for Statistical Computing, Vienna, Austria. 2013. http://www.R-project.org/

TZOULAS, K.; KORPELA, K.; VENN, S.; YLIPELKONEN, V.; KAZMIERCZAK, A.; NIEMELA, J.; JAMES, P. Promoting ecosystem and human health in urban areas using Green Infrastructure: a literature review. Landscape and Urban Planning, v. 81, p. 167-178, 2007. https://doi.org/10.1016/j.landurbplan.2007.02.001 UN - United Nations. World Urbanization Prospects 2018 - Country Profiles: Brazil. United Nations, Department of Economics and Social Affairs, Population Dynamics, 2018. Disponível em: <https://esa.un.org/unpd/wup/CountryProfiles>. Acesso em 26 mai. 2018.

WHITFORD, V; ENNOS, A.R.; HANDLEY, J.F. City form and natural process: indicators for the ecological performance of urban areas and their application to Merseyside, UK. Landscape and Urban Planning, v. 57, p. 91-103, 2001. https://doi.org/10.1016/S0169-2046(01)00192-X

ZHANG, Y.; LIU, Y.; PAN, J.; ZHANG, Y.; LIU, D.; CHEN, J. W.; ZHANG, Z.; LIU, Y. Exploring Spatially Non-Stationary and Scale-Dependent Responses of Ecosystem Services to Urbanization in Wuhan, China. International Journal of Environmental Research and Public Health, vol. 17, 2989, 2020. https://doi.org/10.3390/ijerph17092989

\section{CONTRIBUIÇÃO DOS AUTORES}

Antônio Carlos, Sarah L., Estêvão P., Matheus L. e Rebeca B. planejaram o estudo, coletaram e analisaram os dados e redigiram o texto. Renata Campos orientou todo o desenvolvimento do trabalho, principalmente a etapa de análise de dados, e contribuiu na redação do texto final. Pollyana C. contribuiu na revisão de literatura e redação do texto final. 